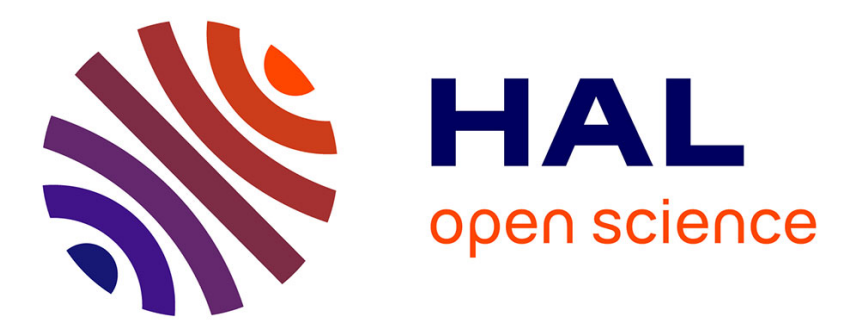

\title{
Control of Complex Robotic Systems: Challenges, Design and Experiments
}

\author{
Ahmed Chemori
}

\section{To cite this version:}

Ahmed Chemori. Control of Complex Robotic Systems: Challenges, Design and Experiments. MMAR: Methods and Models in Automation and Robotics, Aug 2017, Miedzyzdroje, Poland. pp.622631, 10.1109/MMAR.2017.8046900 . lirmm-01718226

\section{HAL Id: lirmm-01718226 https://hal-lirmm.ccsd.cnrs.fr/lirmm-01718226}

Submitted on 27 Feb 2018

HAL is a multi-disciplinary open access archive for the deposit and dissemination of scientific research documents, whether they are published or not. The documents may come from teaching and research institutions in France or abroad, or from public or private research centers.
L'archive ouverte pluridisciplinaire HAL, est destinée au dépôt et à la diffusion de documents scientifiques de niveau recherche, publiés ou non, émanant des établissements d'enseignement et de recherche français ou étrangers, des laboratoires publics ou privés. 


\title{
Control of Complex Robotic Systems: Challenges, Design and Experiments
}

\author{
Ahmed Chemori \\ LIRMM, Univ. Montpellier - CNRS \\ 161 rue Ada, 34095 Montpellier, France \\ Email: Ahmed.Chemori@lirmm.fr
}

\begin{abstract}
Control of robotic systems is a thoroughly investigated subject in the literature, but still an open problem because of the highly nonlinear behaviour of robots, the complexity of their dynamics, the under-actuation, the actuation redundancy, the singularities, kinematic constraints, real-time constraints and more challenges issues. This paper is dedicated to control of robotic systems. They are more and more complex, motivating the development of new sophisticated control schemes. The proposed control schemes are illustrated through different real experimental platforms in four chosen research fields in robotics: underactuated robotics, parallel robotics, humanoid robotics and underwater robotics.
\end{abstract}

\section{INTRODUCTION}

Robotics was initially and for a long time guided by industry needs. Indeed, the early years of robotics was largely focused on robot manipulators [1], used mainly for simple and repetitive automation tasks. The first industrial robot manipulator appeared in 1961 and called Unimate; it was used in the assembly lines of General Motors is USA.

The early control systems for robot manipulators were designed to control independently each axis as a Single-InputSingle-Output (SISO) linear system. Linear control theory was initially used in these fist control systems for robots. Indeed, the coupling dynamics between the different axes of the robot were often neglected and the robot model significantly simplified. Beyond these issues, the primary barriers to progress were the high cost of computation, the lack of good sensors, and the lack of fundamental understanding of robot dynamics. Given these barriers, it is not surprising that two factors were the primary drivers in the advancement of robot control in these early years [2].

However, the progress of robotics and automation as well as their associated innovative applications has required the consideration of more and more complex tasks needing high performances. These challenging tasks required a deeply understanding of complex nonlinear dynamics of robots. Besides, it has motivated the development of new theoretical advances in different control fields, which has consequently enabled more sophisticated applications.

Nowadays, robotic control systems are highly advanced, including robot manipulators [1], underwater robots [3], flying robots [4], mobile robots [5], medical robots [6], parallel robots [7], wearable robots [8], humanoid robots [9] and more others.

In this paper the main challenges related to control of robotic systems will be emphasized, and illustrated through four chosen fields, namely: i) underactuated robotics, ii) parallel robotics, iii) humanoid robotics, and iv) underwater robotics. For all these fields, the motivations and the need of developing sophisticated control schemes will be first highlighted. Then some proposed control solutions will be illustrated through real-time experiments on different platforms from the four above mentioned fields of robotics. The experimental results may show the efficiency and robustness of the proposed control schemes.

This paper is organized as follows, section II will be dedicated to the presentation of research challenges in control of robotics systems. In section III the experimental platforms of our demonstrators will be presented and classified in the four above mentioned fields. Section IV will be devoted to the control and experimental results. The paper ends with some concluding remarks.

\section{RESEARCH CHALLENGES IN CONTROL OF ROBOTIC SYSTEMS}

In the sequel the main research challenges in i) underactuated robotics, ii) parallel robotics, iii) humanoid robotics and iv) underwater robotics are addressed.

\section{A. Underactuated robotics}

Underactuated robotic systems are those mechanical systems with less control inputs than generalized coordinates (called also configuration variables or degrees of freedom (DOFs)); consequently some of their DOFs may be unactuated. Indeed, various examples of such systems have been reported in the robotics literature. Basic examples of underactuated systems are the inverted pendula, including the cart-pole inverted pendulum [10], Furuta inverted pendulum [11], the wheeled inverted pendulum [12] and the inertia wheel inverted pendulum [13]. Other examples include, among others, underactuated manipulators [14], gymnast robots [15] [16], underwater vehicles [17], some mobile robots [18] and some aerial vehicles, like the PVTOL (Planar Vertical Takeoff and Landing) [19].

The underactuation has four sources: i) the nature dynamics of the system, like in the case of some aircrafts, spacecrafts and locomotive systems without wheels, ii) decided at the stage of design of the system itself to reduce the cost, the weight or for some practical purposes, iii) after an actuator failure a fully actuated systems becomes underactuated, iv) imposed artificially to create low-order underactuated systems (like the acrobot and the pendubot) to gain insight in control of higherorder underactuated dynamical systems [20]. 
Underactuated mechanical systems are mainly characterized by a dynamic coupling between the actuated and unactuated coordinates. This dynamic coupling is often nonlinear [21], which generally results in non integrable dynamic constraints (second order non-holonomic). Furthermore, they are characterized by an internal dynamics, which is often unstable, leading to non-minimum phase systems.

For all the above mentioned issues, underactuated systems may require new approaches/techniques to design effective control strategies, therefore they constitute a good framework of nonlinear control problems for both theoretical and practical aspects. For these reasons they are attracting more and more attention of researchers from nonlinear control community as well as many research fields in robotics.

\section{B. Parallel robotics}

Parallel Kinematic Manipulators (PKMs) have gained in the last few decades an increased attention within robotics community [7]. This interest has been stimulated by the obvious advantages of PKMs with respect to their serial counterparts, regarding some specific industrial applications requiring simultaneously high speed and high accuracy. Indeed, to exploit their whole potential and capabilities, a long path is still to be covered. In addition to the mechanical design problem, as well as calibration and optimization of the structure, efficient control design plays a crucial role in improving their overall performances [22].

However, PKMs are known for their highly nonlinear dynamics, that increases considerably when operating at high speeds/accelerations [23], leading to mechanical vibrations issues. Furthermore, uncertainties are very present in these systems due to modelling simplifications, the wear of the components and the inherent variations of the operating environment. Moreover, their coupled dynamics, added to the actuation redundancy issue (i.e. more actuators than degrees of freedom, considered in some mechanisms and leading to some internal efforts) give rise to complex and challenging control problems. Indeed, antagonistic control forces are mainly due to the following sources: i) uncertainties in the model geometry, ii) measurement errors, and iii) non-synchronized control of the actuators [24]. In fact geometric uncertainties [25] can be addressed using identification techniques, while measurement errors are directly related to the instrumentation of the robot and should be explicitly considered in the control design. The last item is straightly related to the control strategy itself. Though non-synchronized control might seem not only related to decentralized controllers, model-based controllers could lead as well to internal forces.

Consequently, for all the above mentioned reasons, new approaches/techniques to design effective control strategies should be considered in the design of control strategies for such systems, taking into account all the previously mentioned issues and challenges.

\section{Humanoid robotics}

Since the early days of the humanoid robotics, most of the studies has been focused on two fields. The first one is the study of biped walking control and stability issues targeting the lower-limbs; while considering the upper-limbs as a dummy mass [26]. The second one is the manipulation of objects or other tasks involving upper-limbs motions without walking [27]. This separation has allowed a simpler study of humanoid control. However, trying to combine upper and lower-limbs motion together is not a trivial task. For instance if these two motions are designed separately, stability may not be guaranteed [28].

To tackle this important problem and improve the efficiency of control, the humanoid robot has to be considered as a whole-body system [29]. In the literature, several approaches have been proposed to deal with whole-body control; most of them can be classified into three classes: i) optimizationbased control, ii) human-capture-based control and iii) taskbased control.

Optimization-based control relies on accurate model of the robot and its environment to compute off-line the best joints trajectories. Several studies, such as [30], have used this methodology to produce whole-body motions. However, this method has several drawbacks: i) the optimization problem to be solved can take a very long time to find the optimal solution, ii) an accurate model of the environment is difficult to produce and iii) this approach is not enough reactive, hence difficult to use in a dynamic environment. The optimization can be based on learning algorithms to produce motion without dealing with the model constraints, but learning algorithms may need many iteration to converge to a satisfactory solution.

Human-capture-based control relies on using sensors to record human motion data. Then the recorded data are then used to generate human-like motions such as balancing [31], walking[32], or dancing[33]. However, despite apparent similarities between the human and the humanoid robot, the human locomotor system is much more complex than the robot locomotor. Therefore, a direct mapping from human captured motion to humanoid robot will often fail and even may lead to instable motions. This implies that the data need to be carefully adapted to take into account the specific structure of the robot such as the kinematics, the joint limits, the size, etc.

The above mentioned issues, show clearly the need of developing new approaches/techniques to design effective control strategies in whole-body motion generation/coordination in humanoid robotics.

\section{Underwater robotics}

Nowadays various underwater robots [17] are available in the market or inside research laboratories. Underwater vehicles are designed to suit specific applications and their development is in growth due to the high demand in various fields where they are needed. They are capable of operating in environments considered to be beyond the reach of human divers. Moreover, they can be used in hazardous environments and can operate as long as needed 24 hours a day when tethered. In research, they have gained a widespread interest in the last decades, from various communities (design, actuation, perception, modelling, control, etc), given the multiple tasks they can accomplish. Indeed, their applications are multiple and various in different fields such as dams inspection, oil and gas industry, aquaculture, wind parks, hydroelectric power stations, underwater archeology, ocean cartography, air crash and environmental investigations, etc. 
Control of underwater vehicles [34] [35] is a thoroughly investigated subject in the literature, but still an open research problem. Indeed, when we are interested in autonomous control of underwater vehicles (ROVs, AUVs, ASVs, bioinspired, etc) ${ }^{1}$ different control challenges may arise. These challenges are related to their inherent high nonlinearities and the timevarying behavior of the vehicles dynamics, subject to hydrodynamic effects, uncertainties and external disturbances.

In fact, the underwater environment is often unstructured, non-uniform, and time-varying. This adds a complexity to the control of such systems, since the dynamic model of the vehicle cannot be fully determined given that some of its parameters are hard to compute and are seldom constant (hydrodynamic coefficients, nonlinear damping, etc). Indeed, the model parameters are likely to change with the operational conditions and the mission. For instance, when the robot is required to manipulate objects [3], or carry payloads, or even to be equipped with some additional sensors, its weight changes, as well as its centers of buoyancy and gravity. Other common examples are the change of buoyancy when the water salinity changes, or the damping increase when some algae gets a grip on the vehicle. Trajectory tracking may also involve accounting for some expected or unexpected external disturbances such as waves, common in shallow water, or random obstacles that the vehicle may fail to avoid.

For all the mentioned above challenges, it is desirable to design a controller able to deal with the inherent complex dynamics of the system, while being robust to compensate parameter changes and reject external disturbances.

\section{OUR EXPERIMENTAL PLATFORMS}

\section{A. Underactuated robotics}

For sake of validation of the control schemes in underactuated robotics, the platform of an Inertia Wheel Inverteed Pendulum (IWIP) will be used. The Inertia Wheel Inverted Pendulum is considered as a benchmark of underactuated mechanical systems. The experimental platform of such a system is shown in Fig. 1, including a mechanical part, an electromechanical part and control PC. The mechanical part of the system is displayed in Fig. 2, it consists of a pendulum body equipped with an actuated reaction wheel. The joint between the pendulum body and the frame is unactuated (passive joint) ; however the joint between the pendulum body and the reaction wheel is actuated (active joint) by a DC motor. The pendulum angle (with respect to the vertical) is constrained to evolve within the interval $\left[-10^{\circ},+10^{\circ}\right]$ due to the mechanical stops at both sides of the pendulum body as illustrated in Fig. 2. The actuator of the inertia wheel is equipped with an embedded incremental encoder allowing the measurement of its angular position. The angle of the pendulum is measured by an inclinometer fixed at the body of the pendulum.

\section{B. Parallel robotics}

For sake of validation of the control schemes in parallel robotics, three experimental platforms of redundantly-actuated

\footnotetext{
${ }^{1}$ ROV: Remotelly Operated Vehicles, AUV: Autonomous Underwater Vehi-
} cles, ASV: Autonomous Surface Vehicles.

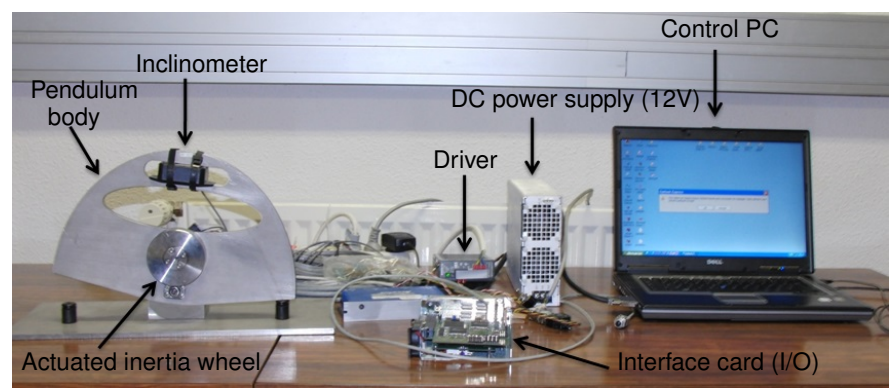

Fig. 1: View of the testbed of the inertia wheel inverted pendulum system.

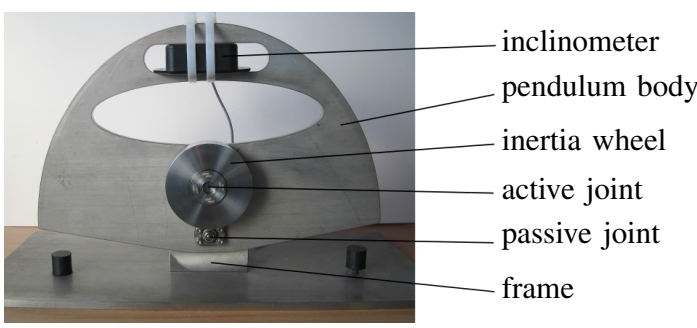

Fig. 2: View of the mechanical part of the inertia wheel inverted pendulum.

(called also over-actuated) PKMs will be used, namely DUAL$\mathrm{V}, \mathrm{R} 4$ and ARROW robots.

1) DUAL-V experimental platform: The testbed of DUAL$\mathrm{V}$ robot is shown in Fig. 3. It is a 3-dof planar redundantly actuated PKM developed at LIRMM laboratory (France), within the framework of a collaboration with the University of Twente (Netherlands). DUAL-V belongs to the 4-RRR family in which, every RRR chain is composed of an actuator, a crank and a coupler. The manipulators links are made with aluminum and the arrangement of the four RRR chains allows one rotation and two translations of its moving platform. Hence, the position of its moving platform is described by two translations and one rotation.

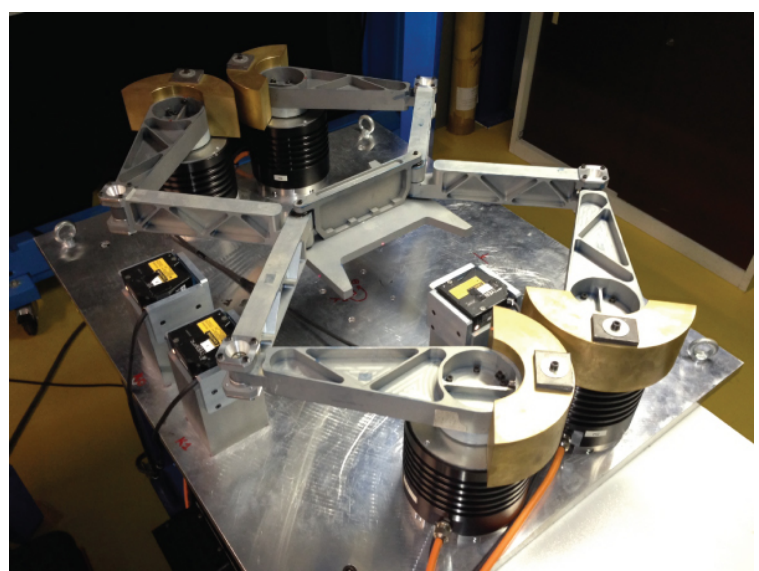

Fig. 3: View of the experimental setup of the redundantlyactuated DUAL-V parallel robot. 
2) R4 experimental platform: Illustrated in Fig. 4, R4 is a redundantly actuated parallel manipulator, which has three DOFs and four actuators. Its degrees of freedom consist in three Cartesian translations and its actuators are the TMB0140-100-3RBS ETEL direct-drive motors which can generate a maximum torque of $127 \mathrm{~N} . \mathrm{m}$ and a maximum speed of $550 \mathrm{rpm}$. The workspace of the robot is equivalent to a cylinder of $300 \mathrm{~mm}$ radius and $100 \mathrm{~mm}$ height.

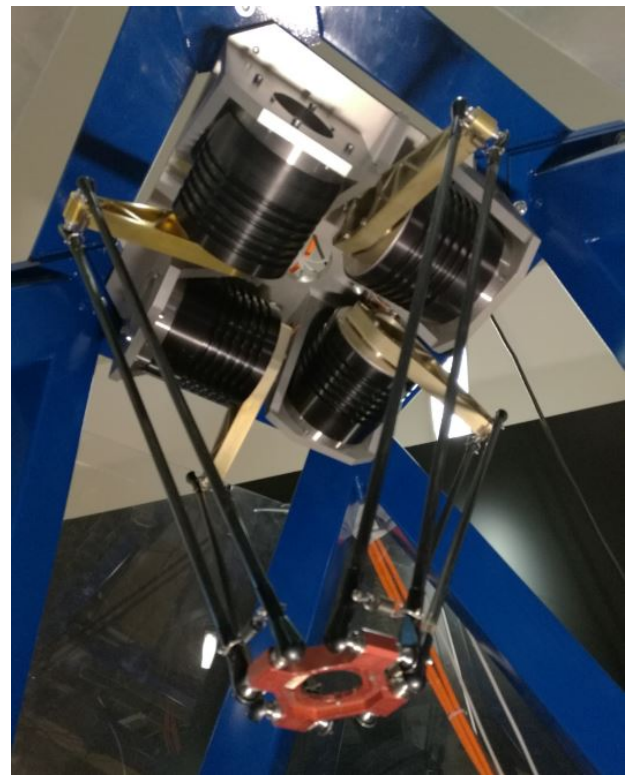

Fig. 4: View of the experimental setup of the redundantly actuated R4 parallel robot.

3) ARROW experimental platform: The ARROW robot is a redundantly actuated parallel manipulator (displayed in Fig. 5) developed at our laboratory within a national project called also ARROW (Accurate and Rapid Robots with large Operational Workspace). Along with its 1-DOF rotational turntable, the entire structure has five DOFs. The parallel module of the robot consists of six linear actuators linked to a moving platform having four DOFs. Thus, the allowed motion for the moving platform is of 3T-1R type; three spatial translations and one rotation along the vertical axis parallel to the fixed-base. The parallel module has two degrees of actuation redundancy, while the turntable has one. Consequently, the entire machine has three degrees of actuation redundancy; eight actuators (six of them are linear while the remaining two are rotational). From a control point of view, the most relevant part to be controlled is the parallel module.

\section{Humanoid robotics}

In humanoid robotics, two experimental platforms are available at our laboratory (http://www.lirmm.fr) for the validation of the developed control schemes, they are presented in the following.

1) SHERPA biped robot: The first robot is SHERPA, a two-leg biped walking robot fully designed in our laboratory. Illustrated in Fig. 6, SHERPA has been developed within the framework of the national project ANR-06-BLAN-0244, called

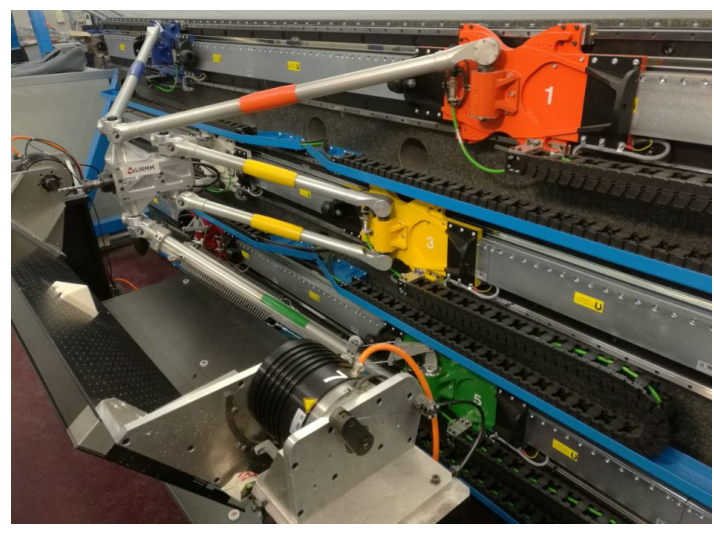

Fig. 5: View of the experimental setup of the redundantly actuated ARROW parallel robot.

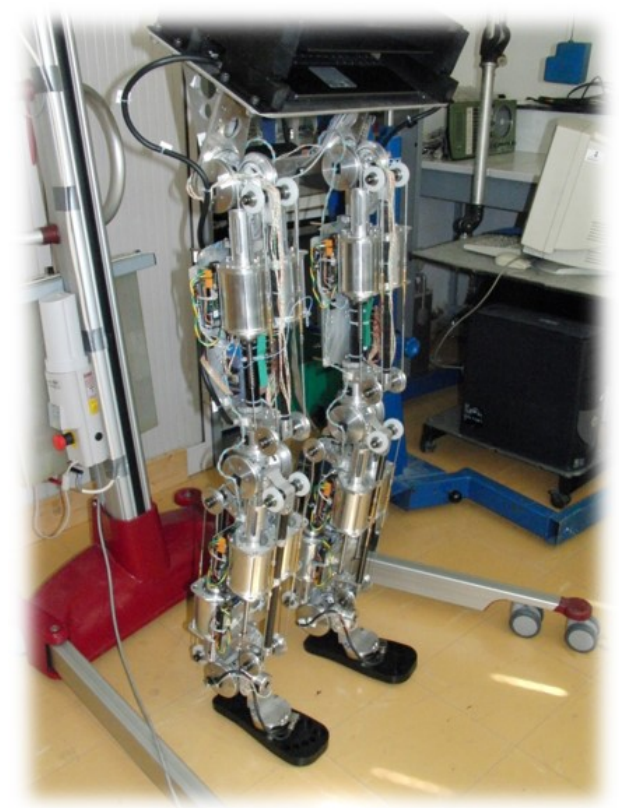

Fig. 6: View of the experimental setup of SHERPA walking robot.

also SHERPA. The robot SHERPA was designed to carry loads while walking in a human environment. This fact constitutes the reason behind its name "Sherpa", meaning people from Tibetan living in the Nepalese Himalayas, who serve as porters on mountain climbing expeditions. This robot is $120 \mathrm{~cm}$ tall, $60 \mathrm{~kg}$ weight, and has 12 DOFs. Each leg has six degrees of freedom, distributed as follows: three DOFs at the hip, one DOF at the knee and two DOFs at the ankle, which is enough, according to biomechanical studies, to reproduce a human walking gait.

The robot is equipped with absolute optical encoders at all the joints, a three-axis accelerometer and three-axis gyroscope used for posture measurement. The contact forces with the ground are measured with a six-axis force sensor located in the robot's feet. All the six DOFs per leg are actuated by homemade brushless backdrivable motors (i.e. low inertia and low 
friction). These actuators are controlled by an embedded PC running under RTX, a real-time extension for Windows, at a sample frequency of $200 \mathrm{~Hz}$.

2) HOAP3 humanoid robot: The second experimental platform for control in humanoid robotics is the robot HOAP-3, a humanoid robot from Fujitsu company. Illustrated in Fig. 7, this humanoid robot is a whole-body testbed. This robot is $60 \mathrm{~cm}$ tall, $8.8 \mathrm{~kg}$, and has 28 DOFs distributed as follows: each leg has six dof (three DOFs at the hip, one DOF at the knee and two DOFs at the ankle). The torso of the robot has one DOF and the neck 3 DOFs. The arms of the robot are composed of $6 \mathrm{DOFs}$, three DOFs at the shoulder, one DOF at the elbow and two DOFs at the hand. The robot is

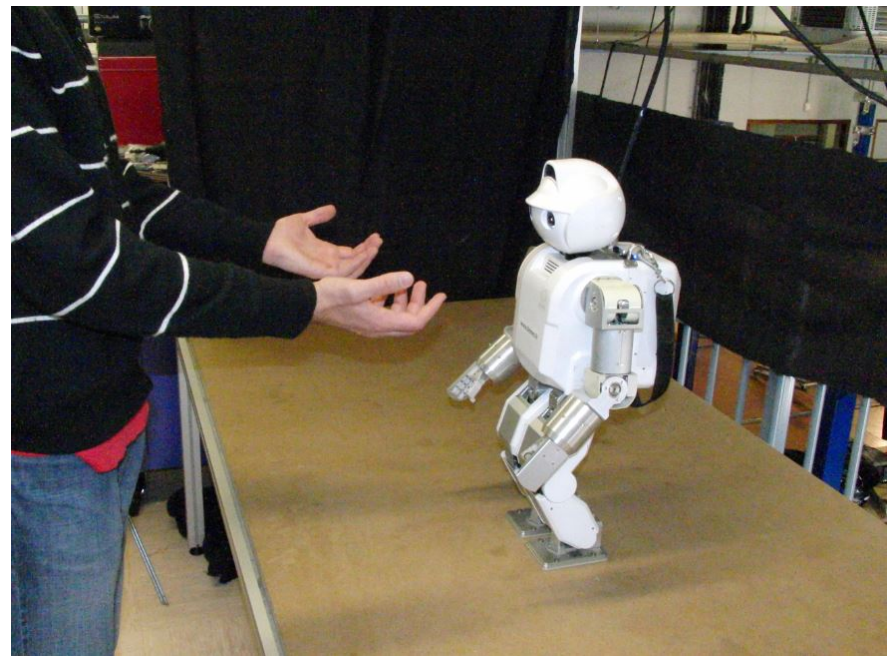

Fig. 7: View of the experimental setup of HOAP3 humanoid robot.

equipped with optical incremental encoders in all the joints to measure in real-time the articular joint positions. A threeaxis accelerometer and three-axis gyroscope are embedded in an IMU to measure the body posture of the robot. Each foot of the robot is equipped with four force sensors to measure the contact forces with the ground. For visual perception of the environment, the robot is equipped with two cameras. The actuators of the robot are brushless motors paired with microcontrollers. The whole system is controlled by an embedded PC running with a real-time kernel RT-Linux at a sample frequency of $1 \mathrm{kHz}$

\section{Underwater robotics}

For underwater robotics, two experimental platforms were uses, namely U-CAT biomimetic AUV and Leonard ROV.

1) U-CAT biomimetic AUV: U-CAT (cf. Fig. 8) is a biomimetic robot designed ${ }^{2}$ specifically to meet the enduser requirements of underwater archaeologists. The design principles of U-CAT are laid down in [36], which describes the constraints and specific requirements posed by the nature of ship penetration in an archaeological mission. As a consequence, a 4-flipper design emerged, not because there was a

${ }^{2} \mathrm{U}-\mathrm{CAT}$ was designed within the framework of ARROWS european project at the center for biorobotics, Tallinn University of Technology in Estonia. specific aim to design a bio-inspired robot but rather because the biomimetic design was most suitable for solving the problem. Particularly, the crucial requirements where the small size and high maneuverability of the vehicles, that required a fully actuated robot with as few as possible actuators. In U-CAT, the 6-DOF actuation (cf. Fig. 9) is achieved using four motors actuating fins. Another requirement was the quiet motion, that was best realized using flippers. As opposed to classical propellers, flippers have a large actuation area and therefore the propelled mass around the robot disturbs less the bottom sediments. This makes close video inspection of a ship interior, for instance, more feasible. The current version of U-

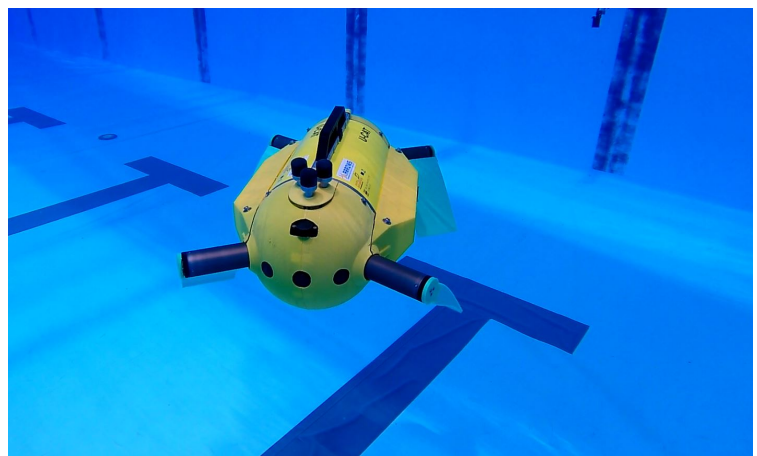

Fig. 8: View of the experimental setup of U-CAT robot.

CAT, illustrated in Fig. 8, is a fully equipped field deployable robotic platform, designed to dive up to $100 \mathrm{~m}$ depth. The 0,6 $\mathrm{m}$ long cylindrical robot houses batteries, electronics, flipper motors and their drivers as well as onboard sensors (IMU, hydrophone array, 8 sonars for obstacle avoidance and a camera), lighting and modem for underwater communication in untethered mode. Fig. 9 shows how the four independently driven flippers are used to achieve maneuverability in 6 DOFs. In addition to the flipper-based control, U-CAT also has a buoyancy control unit that gives an additional means for controlling depth

2) Leonard ROV: The second experimental underwater robotics platform is the robot Leonard, Illustrated in Fig. 10. Leonard is a small tethered underwater remotely operated vehicle. The robot has a size of $75 \mathrm{~cm} \times 55 \mathrm{~cm} \times 45 \mathrm{~cm}$. Its propulsion system includes six thrusters, oriented as illustrated in Fig. 11. With this configuration of the thrusters, all the six degrees of freedom of the robot are actuated and can be controlled. Following the SNAME notation, translational motions are represented by surge, sway, and heave; the rotational motions are represented by roll, pitch, and yaw. According to the illustration of Fig. 11, the surge and the yaw are actuated by the forces $T_{4}$ and $T_{5}$ and the sway is actuated by the force $T_{6}$. The heave, the roll and the pitch are actuated by the forces $T_{1}, T_{2}$ and $T_{3}$. The experimental platform includes the ROV itself driven by a laptop computer, equipped with and Intel Core i7-3520M $2.9 \mathrm{GHz} \mathrm{CPU}$ and $8 \mathrm{~GB}$ of memory. The hardware is running under Windows 7 OS and the control software is developed with Visual $\mathrm{C}++$ language. The robot is equipped with a pressure sensor to measure the depth, an IMU (Inertial Measurement Unite) to measure the robot attitude, and a stereovision system for visual feedback. The actuators of the vehicle are controlled by MD03 Motor Drives. 

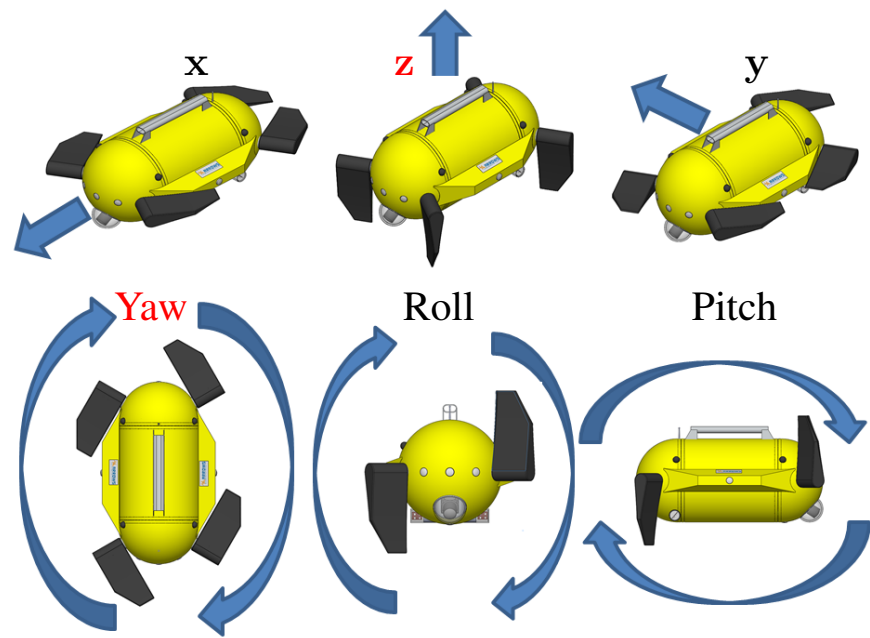

Pitch

Fig. 9: Illustration of how the 4 independently driven flippers are used to actuate the 6 DOFs of the robot separately. The DOFs in red are the subject of the actual study regarding control (i.e. depth and yaw).

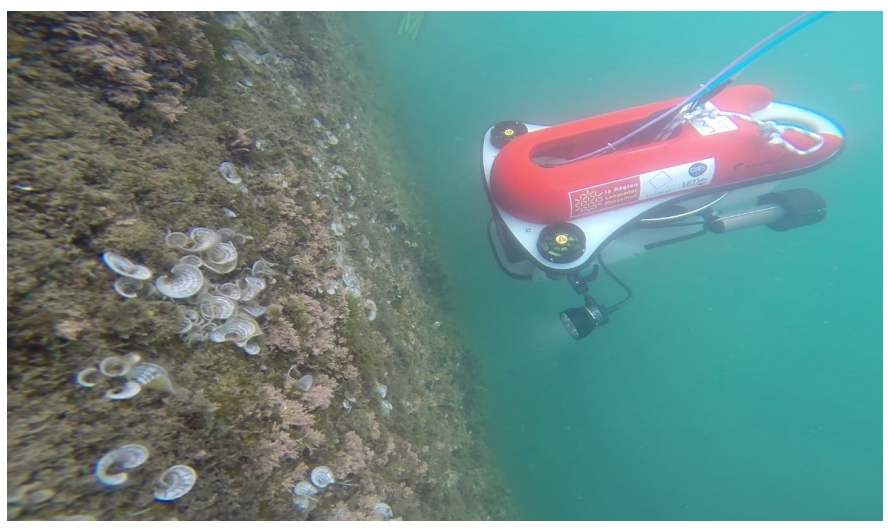

Fig. 10: View of the experimental setup of Leonard ROV.

\section{CONTROL AND EXPERIMENTAL RESUlTS}

\section{A. Underactuated robotics}

The control idea is to find a controller able to stabilized the underactuated system, presented in section III-A, around its unstable equilibrium (inverted pendulum oriented upwards). A robust sliding mode controller is proposed, with a careful attention to the stabilization of the internal dynamics of the system, through a transformation used to write its dynamics in a strict-feedback form [20]. Two experimental scenarios have been performed: i) Stabilisation in the nominal case, and ii) Stabilization with external disturbance rejection.

1) Scenario 1: Stabilization in the nominal case: For this scenario, no external disturbances have been considered. The obtained results for this scenario are depicted in Fig. 12, representing the behaviour of the controlled system. Figure 12-(a) depicts the evolution versus time of the pendulum angle with respect to the vertical. Its velocity is depicted in Fig. 12(b), where we can notice the noise effect due to the numerical derivative. The pendulum is stabilized in less than 5 sec. The

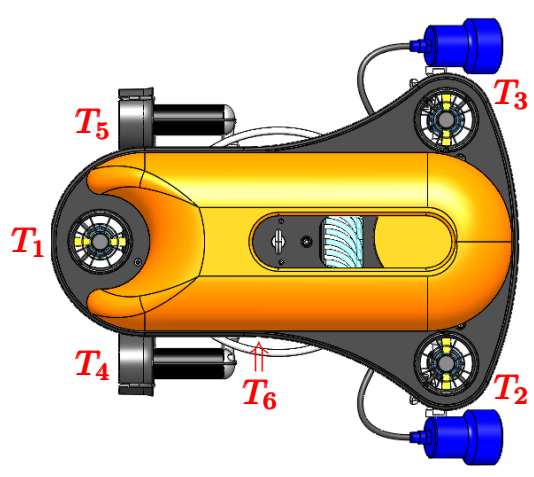

Fig. 11: Illustration of the forces generated by the thrusters of the robot Leonard ROV.

evolution of the velocity of the reaction wheel is displayed in Fig. 12-(c), where we can notice its oscillation around zero. The control input, generated by the proposed controller is depicted in Fig. 12-(d), representing the voltage applied to the actuator of the system.

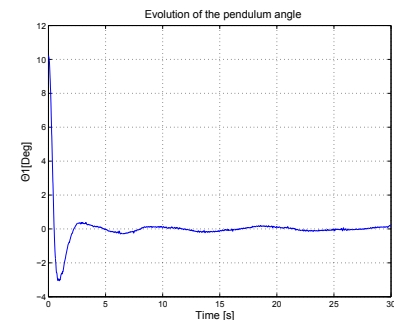

(a) pendulum axis position.

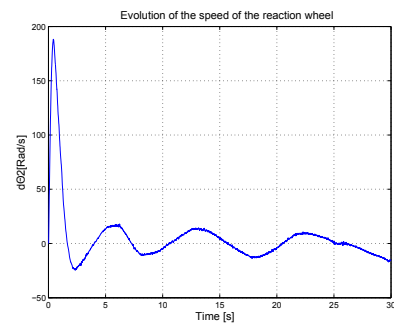

(c) inertia wheel rotation speed.

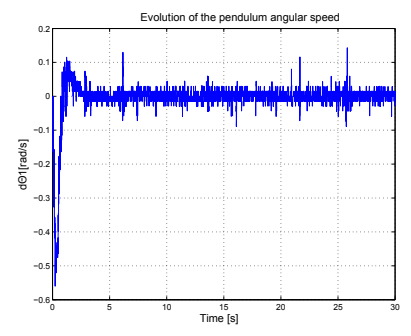

(b) pendulum axis velocity.

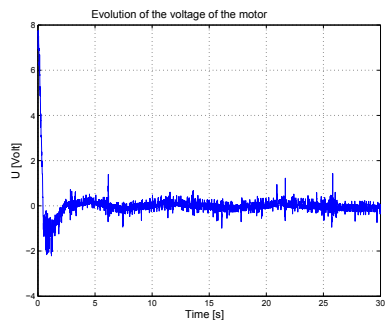

(d) motor driver input.
Fig. 12: Experimental results of the stabilization of the inertia wheel inverted pendulum in the nominal case (scenario 1).

2) Scenario 2: External disturbances rejection: For this scenario, the idea is to consider some external disturbances affecting the system, to test the ability of the proposed controlled in terms of disturbance rejection. As illustrated in Fig. 13, the external disturbances results in external forces applied on the body of the pendulum, generating disturbing torques on the unactuated coordinate of the system (pendulum angle). The obtained results for this scenario are depicted in Fig. 14. The position and velocity of the pendulum are displayed in Figs. 14-(a) and 14-(b), respectively. One can observe the effect of the external disturbance and their rejection by the controller. 


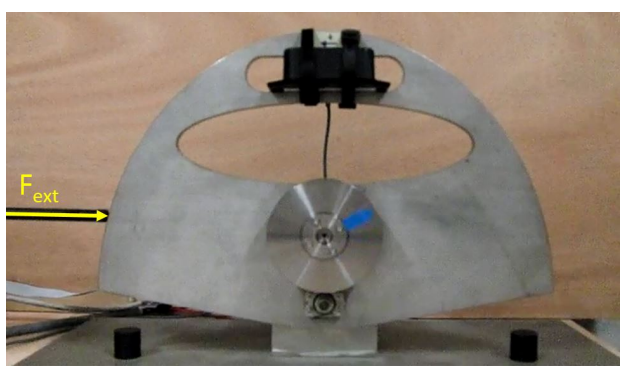

Fig. 13: Illustration of the external disturbance scenario.

We can also notice the effects of these external disturbances on both the speed of the reaction wheel in Fig. 14-(c), as well as in the control input of Fig. 14-(d).

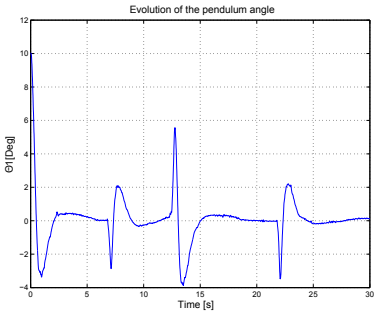

(a) pendulum axis position

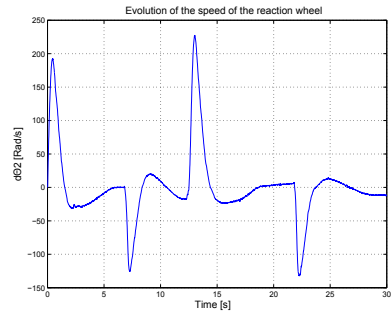

(c) inertia wheel rotation speed.

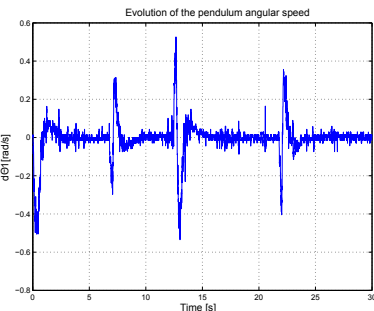

(b) pendulum axis velocity.

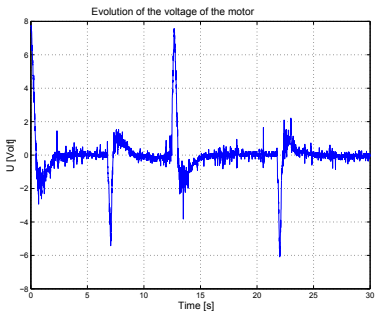

(d) motor driver input.
Fig. 14: Experimental results in the case of external disturbance rejection (scenario 2).

\section{B. Parallel robotics}

In this section some experimental results obtained on the redundantly actuated R4 PKM are presented and discussed. The proposed controllers presented in [23] have been implemented on the experimental platform of the robot, namely a dual-space feedforward and an adaptive dual-space feedforward controllers. Let us here study the convergence of the estimated parameters in the adaptive controller. In other words the effect of the richness of the reference trajectories on the convergence of the estimated parameters will be analyzed. According to [37], the richness of a reference signal is the range of frequencies that it contains, and the necessary and sufficient condition for the reference input (desired trajectories in our case) to result in convergence of the parameter error to zero is that its spectrum must be concentrated on $k \geq N$ lines, where $N$ is the number of unknown parameters in the adaptive scheme. The reference trajectory is a 3D pick-andplace that was computed from a fifth order equation which guaranteed smoothness of the desired positions, velocities and accelerations. The fifth order equation is enough rich to provide a notably fast convergence of the estimated parameters. The convergence of the estimated parameters (mass $\hat{M}_{t o t}$ and inertia $\hat{I}_{t o t}$ of the travelling plate of the robot including eventual payloads) for the 3D pick-and-place trajectory is displayed in Figs. 15 and 16.
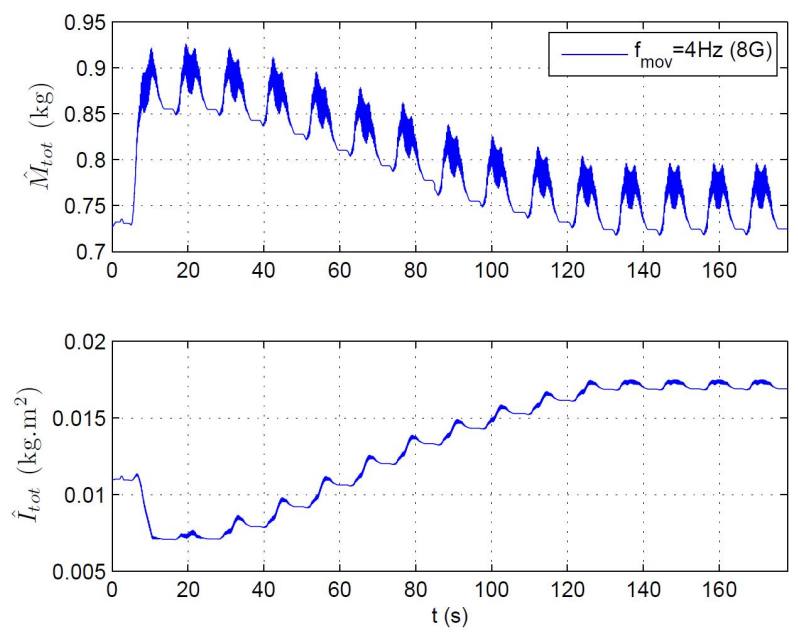

Fig. 15: Evolution of the estimated parameters $\hat{M}_{t o t}$ and $\hat{I}_{t o t}$ for $f_{m o v}=4 \mathrm{~Hz}(8 \mathrm{G})$ with a payload of $200 \mathrm{~g}$.
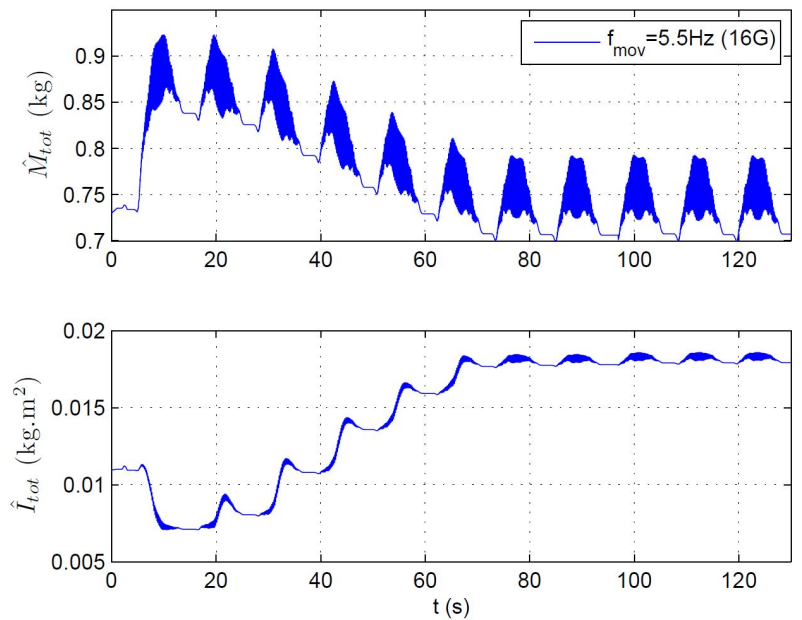

Fig. 16: Evolution of the estimated parameters $\hat{M}_{t o t}$ and $\hat{I}_{t o t}$ for $f_{m o v}=5.5 \mathrm{~Hz}(16 \mathrm{G})$ with a payload of $200 \mathrm{~g}$.

Indeed, the convergence of the estimated parameters for a movement with A frequency $f_{m o v}=4 \mathrm{~Hz}(8 \mathrm{G})$ and for a movement with $f_{m o v}=5.5 \mathrm{~Hz}(16 \mathrm{G})$ with a payload of $200 \mathrm{~g}$ is analyzed. For $8 \mathrm{G}$ of acceleration, the convergence took around 130 seconds to be accomplished (i.e. approximately 11 cycles of the reference trajectory), while for $16 \mathrm{G}$, the convergence is much faster with only around 70 seconds (approximately 6 cycles). 


\section{Humanoid robotics}

To control the two humanoid robots presented in section III-C, the whole-body controller proposed in our previous work [38] has been implemented on both experimental platforms. The proposed control solution is a whole-body control architecture including a ZMP regulation to ensure dynamic stability. The resulting architecture is then hybride, since it includes kinematic control (based on task formalism) as well as dynamic control (based on ZMP feedback and regulation).

1) Control of squat-like motion on SHERPA robot: The proposed control scheme has been implemented on SHERPA biped robot for squat-like motions. The desired relative feet pose is considered constant. The desired CoM (Center of Mass) position moves up and down in a sinusoidal way to produce a simple squat-like motion as illustrated in Fig. 17. The obtained experimental results for this scenario are depicted
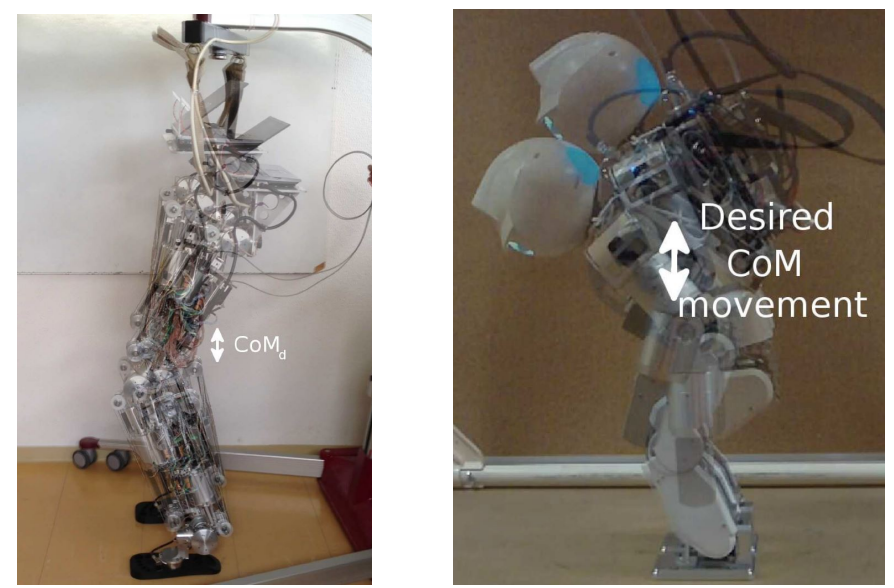

Fig. 17: Illustration of the experimental scenario for both robots (SHERPA and HOAP3).

in Figs. 18 and 19. The evolution of the joint trajectories is displayed in Fig. 18. During the period 5 $-10 \mathrm{~s}$, the robot motion is interpolated from the straight position to the ready position. From $t=10$ to $t=15 \mathrm{~s}$, the control scheme is applied without motion, this allow a convergence to the comfort position. After $t=15 \mathrm{~s}$ seconds, the desired motions are applied. The generated trajectories converge to a periodic motion since all objectives are periodic. It is worth to note, from Fig. 18, that some degrees of freedom are not solicited by the control scheme, since not useful for the desired objectives. In Fig. 19, the amplitude of the measured contact forces with the ground are displayed. The left and the right foot contact forces amplitudes are labeled $\mathrm{W}$ left and $\mathrm{W}$ right respectively. The position of the ZMP under the sole are labeled $x$ and $y$ for the frontal and lateral position relatively to the center of each foot. The obtained squat-like motion is stable thanks to the ZMP regulation loop in the proposed control scheme.

2) Control of Squat-like motion on HOAP3: The objective of this experimental scenario is to produce a whole-body control on the HOAP3 humanoid robot by moving the CoM position up and down according to a sinusoidal signal as illustrated in Fig. 17. The desired relative feet pose is set to be constant for this scenario. The evolution of the joint trajectories

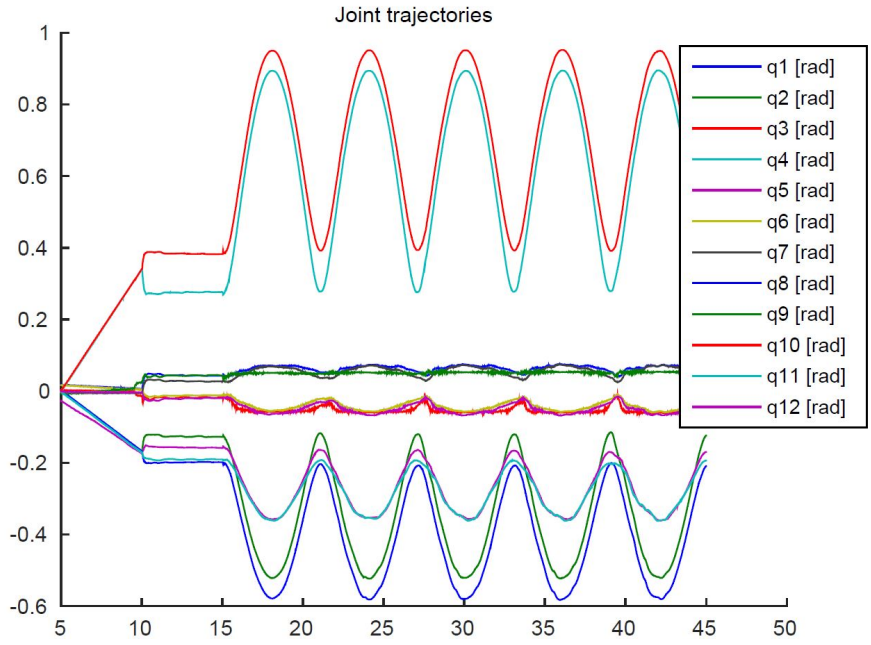

Fig. 18: Evolution of the joint angles of the robot SHERPA.

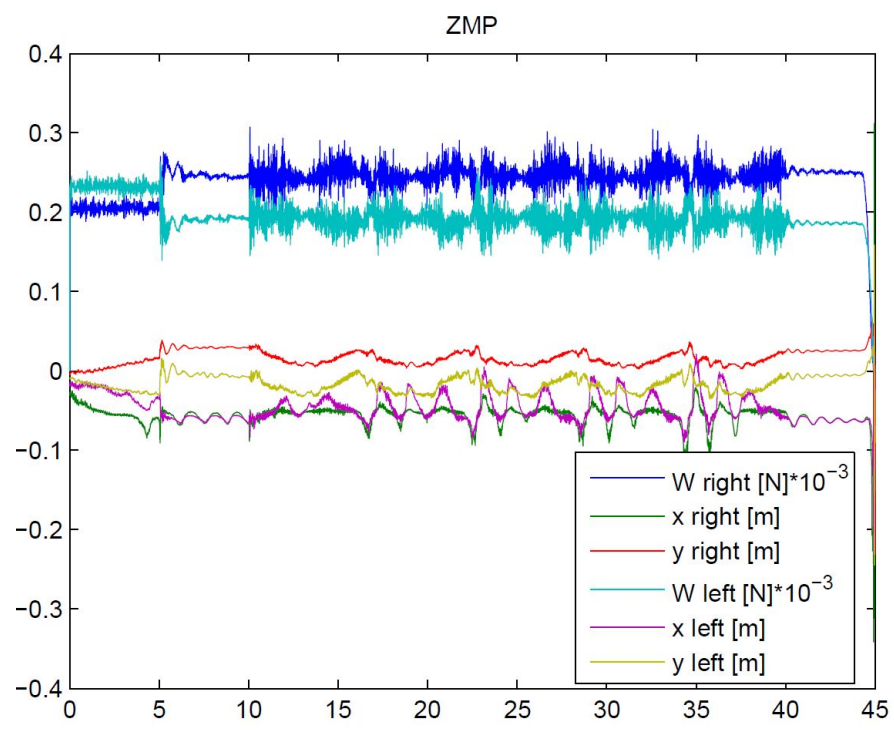

Fig. 19: Evolution of the ZMP measurements on SHERPA biped robot.

are displayed in Fig. 20. These trajectories converge to a periodic motion since the main objective is periodic. According to Fig. 20, this motion uses only some degrees of freedom, enough to reproduce the desired motion. In Fig. 21, the amplitude of the measured contact forces with the ground are displayed. According to the obtained results, one can observe that the produced motions are continuous and smooth, without switching phases, and the robot produces the desired squatlike motion illustrated in Fig. 17, while guaranteeing dynamic stability.

\section{Underwater robotics}

In this section, some of the obtained real-time experimental results in underwater robotics are presented and discussed. Different controllers have been implemented on UCAT biomimetic underwater AUV, hereafter we present some 


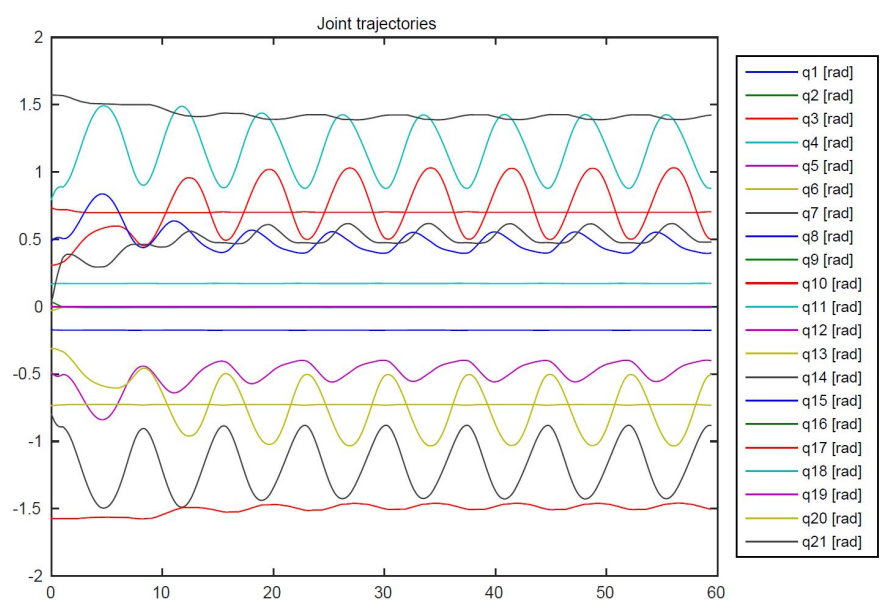

Fig. 20: Evolution of the joint angles of the robot HOAP 3.

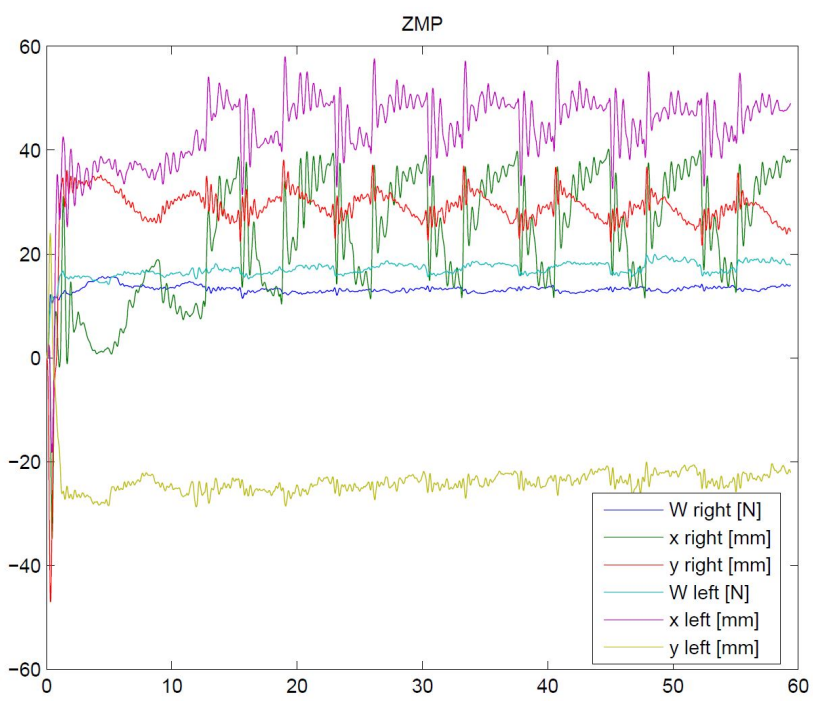

Fig. 21: Evolution of the ZMP measurements on HOAP 3 humanoid robot.

of the obtained results regarding multivariable depth and yaw control. The results of an adaptive inverse dynamics controller (also called nonlinear adaptive state feedback controller) are presented and discussed. This controller provides an online estimation of possible unknown/time-varying model parameters in order to ensure a good trajectory tracking despite the uncertainties and variations in the parameters. Two experimental scenarios have been considered, namely the nominal case and the external disturbance rejection case, presented hereafter.

1) Scenario 1 - Depth and yaw control in nominal case: The idea of this first experimental scenario is to test the proposed adaptive controller in the nominal case. This means that the scenario will be conducted without external disturbances and without changes in the dynamic parameters of the robot. The main objective is to track some predefined time-varying references trajectories on both degrees of freedom (depth and yaw). The obtained results for this experiment are depicted in Fig. 22 displaying the evolution, versus time, of the two controlled degrees of freedom, as well as their associated control inputs. The proposed controller is able to keep the robot around the predefined reference trajectories, and the generated control inputs are not saturated.
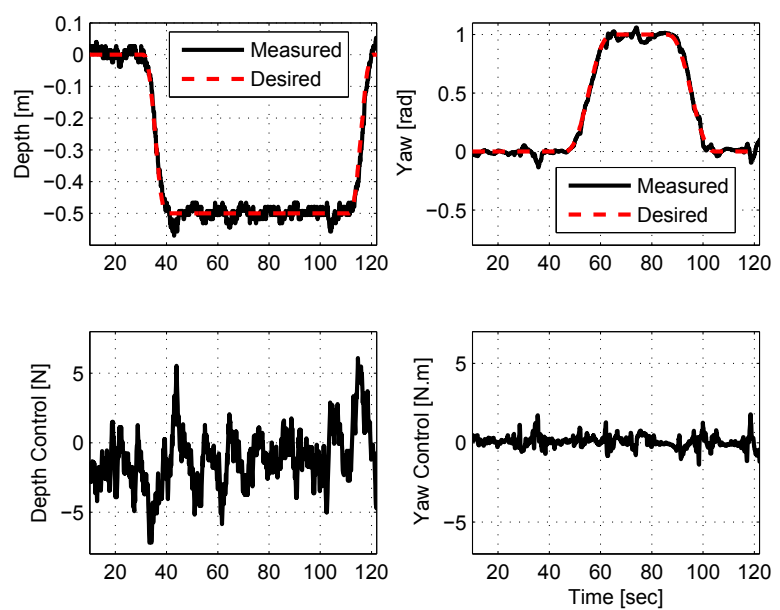

Fig. 22: Obtained experimental results for U-CAT control in scenario 1 : Nominal case.

2) Scenario 2 - Depth and yaw control with external disturbances: The main objective behind this experimental scenario is to test the ability of the proposed controller to reject external disturbances. Indeed, the same predefined timevarying reference trajectories, used for the previous scenario, are proposed to be tacked in the actual scenario. Once the system outputs (depth and yaw) reach the middle steady-state value, an external disturbance is applied. The behaviour of the system is recorded to show the reaction of the controller and its ability to steer back the system's outputs to their reference values. The obtained results are depicted in Fig. 23. According the obtained curves, the adaptive controller is able to reject the applied external disturbance and brings back the outputs around their reference trajectories. The effect of the applied external disturbance is visible on both outputs (depth and yaw).

\section{CONCLUSION}

This paper deals with challenges related to control of complex robotics systems. Indeed, these systems are more and more complex and need more sophisticated control schemes. The research challenges of complex robotic systems have been addressed, and illustrated through four chosen research fields in robotics, namely i) underactuated robotics, ii) parallel robotics, iii) humanoid robotics, and iv) underwater robotics. For all these fields, the motivations and the need of developing sophisticated control schemes have been highlighted. Some proposed control solutions have been validated through realtime experiments on different platforms of the four above mentioned fields of robotics. The experimental results show the effectiveness and robustness of the proposed control schemes. 

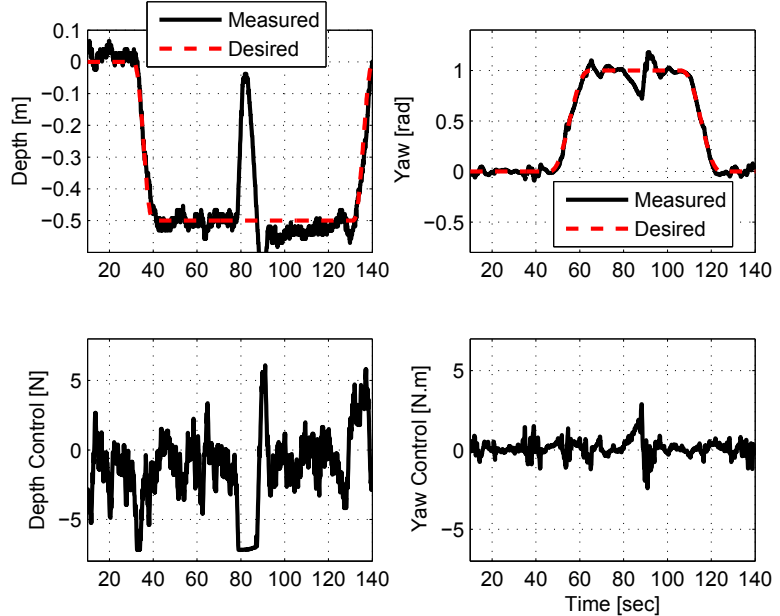

Fig. 23: Obtained experimental results for U-CAT control in scenario 2 : External disturbance rejection.

\section{REFERENCES}

[1] L. Sciavicco and B. Siciliano, Modeling and control of robot manipulators. London, UK: Springer, Second Edition, 2000.

[2] M. Spong and M. Vidyasagar, Robot Dynamics and Control. New York, USA: John Wiley and Sons, 1989.

[3] G. Antonelli, Underwater Robots: Motion and Force Control of VehicleManipulator Systems. Berlin, Germany: Springer, Second Edition, 2006.

[4] K. Nonami, F. Kendoul, S. Suzuki, W. Wang, and D. Nakazawa, Autonomous Flying Robots. Springer, 2010.

[5] S. S. Ge and F. L. Lewis, Autonomous Mobile Robots: Sensing, Control, Decision Making and Applications. Taylor \& Francis, 2006.

[6] A. Schweikard and F. Ernst, Medical robotics. Springer, 2015.

[7] J. Merlet, Parallel Robots. Springer-Verlag, Netherlands, 2006.

[8] J. Gonzalez-Vargas, J. Ibanez, J. L. Contreras-Vidal, H. van der Kooij, and J. Pons, Wearable Robotics: Challenges and Trends. Springer, 2017.

[9] S. Kajita, H. Hirukawa, K. Harada, and K. Yokoi, Introduction to Humanoid Robotics. Springer, 2014.

[10] S. Jadlovska and J. Sarnovsky, "A complex overview of modeling and control of the rotary single inverted pendulum system," POWER ENGINEERING AND ELECTRICAL ENGINEERING, vol. 11, no. 2, pp. 73-85, 2013.

[11] C. Aguilar-Avelar and J. Moreno-Valenzuela, "A composite controller for trajectory tracking applied to the furuta pendulum."

[12] S. Jeong and T. Takahashi, "Wheeled inverted pendiulum type assistant robot : Inverted mobile, standing, and sitting motions," in Proc. IEEE/RSJ Int. Conf. on Intel. Rob. and Systems, San Diego, CA, USA, 2007.

[13] S. Andary, A.Chemori, and S. Krut, "Control of the underactuated inertia wheel inverted pendulum for stable limit cycle generation," $R S J$ Adanced Robotics, vol. 23, no. 15, pp. 1999-2014, 2009.

[14] M. Bergerman, "Dynamics and control of underactuated manipulators," Ph.D. dissertation, Carnegie Mellon University, 1996.

[15] A. D. Luca, R. Mattone, and G. Oriolo, "Stabilization of underactuated planar 2r manipulator," Int. J. Robust and Nonlinear Control, vol. 10, no. 4, pp. 181-198, 2000.

[16] M. Spong, "The swing up control problem for the acrobot," IEEE Control Systems Magazine, vol. 15, no. 1, pp. 49- 55, February 1995.

[17] T. Fossen, Marine Control Systems:Guidance, Navigation and Control of Ships, Rigs and Underwater Vehicles. Trondheim, Norway: Marine Cybernetics, 2002.
[18] S. Ge and F. Lewis, Autonomous Mobile Robots. CRC Press, 2006.

[19] G. Poulin, A. Chemori, and N. Marchand, "Minimum energy oriented global stabilizing control of the PVTOL aircraft," Inter. Jour. of Cont., vol. 80, no. 3, pp. 430-442, 2007.

[20] R. Olfati-Saber, "Nonlinear control of underactuated mechanical systems with application to robotics and aerospace vehicles," Ph.D. dissertation, Massachusetts Institute of Technology, 2001.

[21] H. Khalil, Nonlinear Systems. NJ, USA: Prentice Hall, Upper Saddle River, Second Edition.

[22] M. Bennehar, A. Chemori, M. Bouri, J. L. F., and F. Pierrot, "A new rise-based adaptive control of pkms: Design, stability analysis and experiments," International Journal of Control, vol. DOI: 10.1080/00207179.2017.1286536, 2017.

[23] G. Sartori-Natal, A. Chemori, and F. Pierrot, "Dual-space control of extremely fast parallel manipulators: Payload changes and the $100 \mathrm{~g}$ experiment," IEEE Transactions on on Control Systems Technology, vol. 23, no. 4, pp. 1250-1535, 2015.

[24] A. Muller, "Effects of geometric imperfections to the control of redundantly actuated parallel manipulators," in In IEEE International Conference on Robotics and Automation (ICRA'09), Kobe, Japan, 2009.

[25] _ - "Consequences of geometric imperfections for the control of redundantly actuated parallel manipulators," IEEE Transactions on Robotics, vol. 26, no. 1, pp. 21-31, 2010.

[26] M. H. Raibert, "Legged robots," vol. 29, pp. 499-514, 1986.

[27] T. Asfour, P. Azad, N. Vahrenkamp, K. Regenstein, A. Bierbaum, K. Welke, J. Schroeder, and R. Dillmann, "Toward humanoid manipulation in human-centred environments," Robotics and Autonomous Systems, vol. 56, no. 1, pp. 54-65, 2008.

[28] S. Nakaoka, A. Nakazawa, K. Yokoi, H. Hirukawa, and K. Ikeuchi, "Generating whole body motions for a biped humanoid robot from captured human dances," in Proceedings of the IEEE International Conference on Robotics and Automation ICRA'03, Seoul, Korea, 2003, pp. 3905-3910.

[29] O. Khatib, L. Sentis, and J.-H. Park, "A unified framework for wholebody humanoid robot control with multiple constraints and contacts," in in European Robotics Symposium, 2008, pp. 303-312.

[30] S. Lengagne, N. Ramdani, and P. Fraisse, "Guaranteed computation of constraints for safe path planning," in in Proceedings of IEEE International Conference on Humanoid Robots (Humanoids07), 2007, pp. 312-317.

[31] F.-J. Montecillo-Puente, M. N. Sreenivasa, and J.-P. Laumond, "On real-time whole-body human to humanoid motion transfer," in in Proceedings of International Conference on Informatics in Control, Automation and Robotics (ICINCO10), 2010, pp. 22-31.

[32] S. Kim, C. Kim, and S. You, B.and Oh, "Stable whole-body motion generation for humanoid robots to imitate human motions," in in Proceedings of IEEE International Conference on Intelligent Robots and Systems (IROSO9), 2009, pp. 2518-2524.

[33] K. Yamane and J. Hodgins, "Simultaneous tracking and balancing of humanoid robots for imitating human motion capture data," in in Proceedings of IEEE International Conference on Intelligent Robots and Systems (IROSO9), 2009, pp. 2510-2517.

[34] D. Maalouf, A. Chemori, and Creuze, "L1 adaptive depth and pitch control of an underwater vehicle with real-time experiments," Ocean Engineering, vol. 98, pp. 66-77, 2015.

[35] E. Campos, A. Chemori, V. Creuze, J. Torres, and R. Lozano, "Saturation based nonlinear depth and yaw control of underwater vehicles with stability analysis and real-time experiments," Mechatronics, vol. 45, pp. 49-59, 2017.

[36] T. Salumae, R. Raag, J. Rebane, A. Ernits, G. Toming, M. Ratas, and M. Kruusmaa, "The arrows project: adapting and developing robotics technologies for underwater archaeology," in IEEE Oceans-St. John's, 2014.

[37] S. Boyd and S. Sastry, "Necessary and sufficient conditions for parameter convergence in adaptive control," Automatica, vol. 22, no. 6, pp. 629-639, 1986

[38] D. Galdeano, V. Bonnet, M. Bennehar, P. Fraisse, and A. Chemori, "Partial human data in design of human-like walking control in humanoid robotics," in in 10th International IFAC Symposium on Robot Control (SYROCO12), Dubrovnik, Croatia, 2012, pp. 485-490. 